\title{
Basic amino acids in fodder and bitter biotypes of Lupinus angustifolius *
}

H. BIRECKA, E. NALBORCZYK

\section{INTRODUCTION}

For more than fifteen years (Sch öpf 1949, 1959) the attention of many workers has been paid to lysine as the main precursor of lupin alkaloids (Mothes 1955; Hegnauer 1958; Schütte, Nowacki 1959; 1962, Schütte 1960, Schütte, Schäfer 1961, Schütte et al. 1961 a, b, 1962, Nowacki, Byerrum 1962, Hasse, Schmid 1963 a, b, Luckner 1964, Schütte, Hindorf 1964).

Various hypotheses concerning possible transformations of this amino acid into spartein skeleton have been discussed in detail by Schütte et al. (1964). These hypotheses are mainly based on results obtained in investigations on the character and degree of incorporation - into alkaloids - of labelled lysine or cadaverine after their introduction into lupin plants. It is assumed that one alkaloid (of sparteine skeleton) molecule is formed by three lysine molecules.

Some other authors suggest another possibility of alkaloid biosynthesis.

Having found much more free arginine in seeds of fodder varieties of L. angustifolius and albus than in those of bitter ones Przybylska (1959) made a supposition that arginine might also be a precursor of alkaloids. Later, Nowacki, Przybylska and Hurich (1960) postulated that arginine and lupin alkaloids might have a common precursor; in fodder lupins, in which its transformation into alkaloids is inhibited, it gives rise to arginine.

The same hypothesis is advanced by Wiewiórowski and Reifer (1961), who 'under 'arginine' understand these precursors, which give rise to arginine in the sweet lupins and to the alkaloidal bases in the bitter ones". They point also at the relatively high content of free arginine in seeds of fodder varieties of L: albus and angustifolius (Wiewiór ows ki and al. 1959). It has to be mentioned that Mazuś and Reifer (1964) - after having introduced ${ }^{14} \mathrm{C}$-arginine into 9-14 days old plants of L. angustifolius - did not find any higher degree of ${ }^{14} \mathrm{C}$ incorporation into alkaloids than that obtained when ${ }^{14} \mathrm{C}$-labelled carbonate was used; therefore they conclude, that arginine cannot be considered as a direct precursor in the biosynthesis of lupin alkaloids.

The lysine and the 'arginine' hypotheses differ significantly from each other and at the same time the experimental argumentation used in both of them may

* Presented at the third International Symposium on "Biochemistry and Physiology of Alkaloids" held in Halle in June 1965. 
cause some strictures, namely: 1) as regards the first one - it must be borne in mind that the kind of transformations undergone by a compound introduced into a plant may or may not reveal its true metabolic pathway in situ. As normal metabolic changes of various organic compounds are in most cases strictly localized not only within one organ but also within one cell, the transformation of an introduced compound may be of a casual character or may reflect only one of various metabolic alternatives, not necessary the main one (the time factor plays also an important role). We pointed at this possibility discussing results concerning alkaloid interconversion in lupin plants (Birecka et al. 1960, Birecka 1963). It should not be underestimated when alkaloid biosynthesis is under consideration; the sites of alkaloid concentration in lupin tissues' (White, Spencer 1964) indicate that their synthesis is rather strictly localized; 2) as regards the 'arginine' hypothesis it must be borne in mind that the conclusion were drawn mainly on the basis of differences in free amino acids in lupin seeds, which, however, are not the site (in any case the significant site) of alkaloid biosynthesis. Moreover free amino acids constitute only a very small proportion of the total amount of amino acids including those bound in proteins.

Taking account of the aforementioned remarks it seemed useful to approach the problem in another way, i.e. 1) avoiding any interference into plant metabolism, which might bring about artefacts and 2) paying attention to plant organs mainly responsible for alkaloid biosynthesis.

It was presumed that differences in alkaloid synthesis and content between fodder and bitter lupin plants might perhaps be accompanied by some differences in the amino acid (or amino acids) that is involved in alkaloid synthesis.

In the reported here experiments with plants of $L$. angustifolius the main attention was paid to lysine and arginine.

\section{MATERIALS AND METHODS}

Two pairs of homologous genotypes* of L. angustifolius were used. Each pair, represented by one fodder and one bitter homozygote, was selected from the progeny of plants, which were obtained by crossing a bitter and a fodder variety: I. 'Wielkopolski' - 'Obornicki', fodder $\times$ local biotype, bitter; II 'Szybkopędny' - 'Obornicki', fodder $\times$ 'Puławski’ early, bitter. (J. Mikołajczyk 1960; Barbacki et al. 1962).

The plants inoculated with a suitable Rhizobium strain were grown in pot cultures on a mixture of sand and soil. Mineral nutrients applied before sowing as well as during plant growth were analogous to those in previous investigations. In order to enhance alkaloid synthesis the plants at flowering were decapitated (promotion of side shoot formation). After 10 days, on July 7 at 430 p.m. they were exposed

* The authors are greatly indebted to dr. Jan Mikołajczyk for suppling them with seeds of these plants. 
to ${ }^{14} \mathrm{C}$-labelled $\mathrm{CO}_{2}$ for two and a half hours in a plexiglass chamber* under natural illumination.

Four plants $(2 \times 2)$ of each homozygote were sampled immediately after exposure, the remaining ones (also $2 \times 2$ ) were kept in darkness for the following 12 hours and then sampled. The plants were killed at $-60^{\circ} \mathrm{C}$, the main stem separated from leaves (including young side shoots). The frozen plant material was then ground and additionally homogenized in a glass homogenizer.

A. Free amino acids: plant material (leaves as well as stems) was extracted exhaustively with $80 \%$ ethanol, the solvent evaporated** and the residue treated

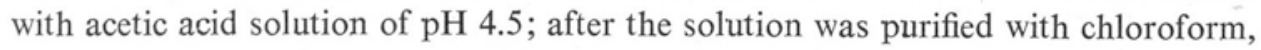
the solvent was evaporated and the residue dissolved in $80 \%$ ethanol. The amino acid content and the radioactivity of the solution were determined.

The amino acids were absorbed quantitatively by Dowex 50 , the resin then was washed with ethanol*** in order to remove some radioactive impurities; amino acids were eluted with $1 \mathrm{~N} \mathrm{NH} \mathrm{NH}_{4} \mathrm{OH}$. After having determined the radioactivity of the solution, alkaloids were removed with chloroform. The solvent was evaporated, the residue dissolved in $1 \mathrm{~N} \mathrm{HCl}$. On a column of Amberlite CG-120 (piridineacetate bufors and $\mathrm{NH}_{4} \mathrm{OH}$ ) the amino acids were divided into three fractions: 1) acid and neutral ones, 2) lysine and histidine and 3) arginine. The first fraction had to be purified once more by means of a small amount of Amberlite CG-120. The two latter were partitioned by means of paper or thin layer chromatography (n-butanol:acetate-water $-45: 5: 12.5$ or n-butanol:5\% $\mathrm{NH}_{4} \mathrm{OH}-4: 1$ ) and then the amount and radioactivity of each amino acid (or of amino acid fraction) were determined.

B. Protein amino acids: the residue - obtained after removing the ethanol extract - was washed with acetone afterwards with ether and then treated with acetic acid solution at $\mathrm{pH} 4.5$, heated on a water bath during $60 \mathrm{~min}$. at $100^{\circ} \mathrm{C}$, cooled and neutralized. In order to eliminate starch the suspension was incubated with diastase for 16 hours at $37^{\circ} \mathrm{C}$ in the presence of toluene and afterwards centrifuged. The supernatant was removed the residue washed and then the proteins hydrolized with $6 \mathrm{~N} \mathrm{HCl}$ at $100^{\circ} \mathrm{C}$. The solvent from the protein amino acid solution was evaporated, then $0,05 \mathrm{~N} \mathrm{HCl}$ was added to the dry residue. The following technical procedures were similar to those applied in analyses on free amino acids.

C. Alkaloids: ethanol from a proportion of the extract, obtained from leaves as well as from stems, was evaporated, alkaloids were extracted quantitatively from the residue with $0.1 \mathrm{~N} \mathrm{HCl}$ (using also chloroform at the end of extraction). The acid solution was alkalized and the investigated bases extracted with chloroform, which after $0.1 \mathrm{~N} \mathrm{HCl}$ was added - was immediately evaporated. The alkaloids were quantitatively determined and then a proportion of them was transferred once more

* This chamber was described in detail in a previous publication (Birecka 1963).

** At about $20^{\circ} \mathrm{C}$ - the same temperature was applied in every case when evaporation was necessary.

*** All rejected solvents were checked. 
into chloroform. After the solvent was removed they were sublimated at $10^{-3} \mathrm{~T}$ at various temperature - between 90 and $170^{\circ} \mathrm{C}$. The amount and radioactivity of each alkaloid fraction* was determined separately. Simultaneously a proportion of purified alkaloid solution was used from chromatographic partition and the specific radioactivity of the investigated bases - after removing hydroxylupanine esters - was determined.

For amino acid determination the spectrometric $(570 \mathrm{~m} \mu)$ method of Moore and Stéin (1948) was used, for alkaloid determination - the method of Reifer and Niziołek (1957).

The radioactivity of plant material as well as of some extracts was determined (using the infinite thin layer technique) by means of a G. M. counter (with a mica window of $1 \mathrm{mg} / \mathrm{cm}^{2}$ ); its efficiency - at the geometry applied was about $5 \%$. The radioactivity of lysine, arginine and alkaloids was determined by means of a $2 \pi$ windowless gas-flow counter (its efficiency was about $35 \%$ ). The data given by the latter were recounted according to the efficiency of the G. M. counter.

In the spring 1965 seeds of $L$. angustifolius, bitter variety 'Wielkopolski gorzki' were sown in sand cultures ( 15 plants per pot) a) without mineral nutrients b) with nutrients normally applied before sowing (the $\mathrm{N}$ dose was increased to $100 \mathrm{mg}$ per pot). The plants were analysed on total alkaloid content before sowing (dry seeds) after imbibition (germinated seeds), 10 and 23 days later (upper green parts and root).

\section{RESULTS}

The two investigated pairs of homozygotes differ from each other, especially when leaf/stem dry weight ratio is taken under consideration. This ratio is higher in 'Wielkopolski' (due mainly to a higher dry weight of leaves) than in 'Szybkopędny' - in fodder as well as in bitter plants (tab. 1). Differences between these two homozygotes - in favor of the bitter one - are more pronounced in 'Wielkopolski' than in 'Szybkopędny'.

The two investigated pairs do not differ significantly from each other as regards the relative (per $g$ d.m.) amounts of the total free and protein amino acids as well as alkaloid content (except for fodder 'Wielkopolski', which shows a higher alkaloid level than the fodder 'Szybkopędny'), However, in 'Wielkopolski' as well as in 'Szybkopędny' the total protein amino acid content in bitter plants, especially in their leaves, is higher than in fodder ones, whereas the total free amino acid content is rather the same (the differences presented in tab. 1 cannot be considered as significant). It should be added that the free amino acids in leaves as well as in stems constitute even less than $10 \%$ of those bound in protein.

The amount of free basic amino acids - lysine and arginine together (histidine was found in traces only) - is extremely small; their share in the total content of free amino acids varies between 1.1 to $3.1 \%$ (less in stems, more in leaves). On the contrary, the share of basic amino acids in proteins is relatively high ranging

* The fractionation of alkaloids was possible only when bitter plants were analyzed. 
from 7.2 to $10.0 \%$ without any significant differences between 'Wielkopolski' and 'Szybkopędny'. As it can be seen from the data presented in table 2 and figs. 1 and 2 there are no significant differences between fodder and bitter plants as regards free lysine or free arginine content. It is worth stressing that the amount of alkaloid $\mathrm{N}$ even in fodder plants exceeds markedly the content of $\alpha$-amino $\mathrm{N}$ of these compounds (in bitter plants 50-80 times!).

Table 1

Amino acid and alkaloid content in fodder and bitter plants of Lupinus angustifolius (per plant)

\begin{tabular}{|c|c|c|c|c|c|c|c|c|c|c|}
\hline \multirow{3}{*}{$\begin{array}{c}\text { Time of } \\
\text { sampling } \\
\text { after exposure } \\
\text { to }{ }^{14} \mathrm{CO}_{2}\end{array}$} & \multirow{3}{*}{ Plant } & \multirow{3}{*}{$\begin{array}{l}\text { Plant } \\
\text { parts }\end{array}$} & \multirow{3}{*}{$\begin{array}{l}\text { Weight } \\
\text { g d.m. }\end{array}$} & \multicolumn{3}{|c|}{$\begin{array}{c}\text { Free amino.acids 1) } \\
\alpha \text {-amino } \mathrm{N}\end{array}$} & \multicolumn{3}{|c|}{$\begin{array}{c}\text { Protein amino acids 1) } \\
\alpha \text {-amino } \mathrm{N}\end{array}$} & \multirow{3}{*}{$\begin{array}{l}\text { Alkaloid } \\
\text { N 4) mg } \\
\text { per g } \\
\text { d.m. }\end{array}$} \\
\hline & & & & \multicolumn{2}{|c|}{ total } & \multirow{2}{*}{$\begin{array}{l}\text { lys. 2) } \\
+ \text { arg. } \\
\text { per cent } \\
\text { of free } \\
\text { amino } \\
\text { acids }\end{array}$} & \multicolumn{2}{|c|}{ total } & \multirow{2}{*}{$\begin{array}{c}\text { lys. } 3 \text { ) } \\
+ \text { arg. } \\
\text { per cent } \\
\text { of prote- } \\
\text { in amino } \\
\text { acids }\end{array}$} & \\
\hline & & & & $\begin{array}{l}\text { mg per } \\
\mathrm{g} \mathrm{d.m}\end{array}$ & mg & & $\begin{array}{l}\text { mg per } \\
\mathrm{g} \mathrm{d.m.}\end{array}$ & $\mathrm{mg}$ & & \\
\hline
\end{tabular}

Wielkopolski

\begin{tabular}{|c|c|c|c|c|c|c|c|c|c|c|}
\hline \multirow{5}{*}{ Immediately } & \multirow[t]{2}{*}{ fodder } & $\begin{array}{l}\text { leaves } \\
\text { stem }\end{array}$ & $\begin{array}{l}3.08 \\
3.91\end{array}$ & $\begin{array}{l}0.7 \\
0.5\end{array}$ & $\begin{array}{l}\left.2.03^{5}\right) \\
2.17\end{array}$ & $\begin{array}{l}3.0 \\
2.2\end{array}$ & 16.1 & 50.5 & 7.7 & $\begin{array}{l}0.025 \\
0.023\end{array}$ \\
\hline & & total & 6.99 & . & 4.20 & & & & & \\
\hline & \multirow{3}{*}{ bitter } & leaves & 3.50 & 1.0 & 3.65 & 1.5 & 22.3 & 77.9 & 7.6 & 0.58 \\
\hline & & stem & 4.51 & 0.5 & 2.94 & -1.1 & & & & 0.35 \\
\hline & & total & 8.01 & & 6.59 & & & & & \\
\hline \multirow{6}{*}{ After 12 hours } & \multirow{3}{*}{ fodder } & leaves & 2.87 & 1.2 & 3.57 & 2.8 & 16.5 & 47.6 & 8.1 & 0.023 \\
\hline & & stem & 3.55 & 0.7 & 2.43 & 2.1 & 6.4 & 22.7 & 7.6 & 0.022 \\
\hline & & total & 6.42 & & 6.00 & & & 70.3 & & \\
\hline & \multirow{3}{*}{ bitter } & leaves & 3.15 & 1.5 & 4.88 & 1.5 & 20.8 & 65.5 & 9.2 & 0.64 \\
\hline & & stem & 4.79 & 0.6 & 3.01 & 1.5 & 7.2 & 34.1 & 7.2 & 0.33 \\
\hline & & total & 7.94 & & 7.89 & & & 99.6 & & \\
\hline
\end{tabular}

Szybkopędny

\begin{tabular}{|c|c|c|c|c|c|c|c|c|c|c|}
\hline \multirow{5}{*}{ Immediately } & \multirow[t]{2}{*}{ fodder } & $\begin{array}{l}\text { leaves } \\
\text { stem }\end{array}$ & $\begin{array}{l}2.20 \\
4.22\end{array}$ & $\begin{array}{l}0.8 \\
0.4\end{array}$ & $\begin{array}{l}1.66 \\
1.55\end{array}$ & $\begin{array}{l}2.9 \\
2.1\end{array}$ & 16.3 & 35.8 & 8.0 & $\begin{array}{l}0.012 \\
0.003\end{array}$ \\
\hline & & total & 6.42 & & 3.21 & & & & & \\
\hline & \multirow{3}{*}{ bitter } & leaves & 2.27 & 1.3 & 2.97 & 1.5 & \multirow[t]{2}{*}{22.5} & \multirow[t]{2}{*}{52.1} & \multirow[t]{2}{*}{7.9} & 0.69 \\
\hline & & stem & 4.62 & 0.7 & 3.10 & 1.1 & & & & 0.27 \\
\hline & & total & 6.89 & & 6.07 & & & & & \\
\hline \multirow{4}{*}{ After 12 hours } & \multirow{3}{*}{ fodder } & leaves & 2.61 & 1.4 & 3.64 & 2.7 & 17.7 & 46.4 & 9.4 & 0.009 \\
\hline & & stem & 3.75 & 0.5 & 2.08 & 2.2 & 6.7 & 25.0 & 7.9 & 0.004 \\
\hline & & total & 6.36 & & 5.72 & & & 71.4 & & \\
\hline & \multirow{3}{*}{ bitter } & leaves & 2.46 & 1.5 & 3.72 & 3.1 & 21.2 & 52.1 & 10.0 & 0.64 \\
\hline \multirow[t]{2}{*}{ - } & & stem & 4.77 & 0.6 & 2.72 & 1.1 & 8.3 & 39.7 & 7.6 & 0.26 \\
\hline & & total & 7.23 & & 6.44 & & & 91.8 & . & \\
\hline
\end{tabular}

1) Lys. - lysine, arg. - arginine.

2) Including traces of histidine (less than $2-3 \%$ of lysine content - see fig. 1).

3) Any traces of histidine neither on paper nor on thin layer chromatograms were found (an amount of histidine greater than $1 \%$ lysine content could have been detected).

4) Assuming that $\mathrm{N}$ content in alkaloids amounts to $11 \%$ of their weight.

5) Only one replication. 
Table 2

Lysine, arginine and alkaloid content in fodder and bitter plants of Lupinus angustifolius (per plant)

\begin{tabular}{|c|c|c|c|c|c|c|c|}
\hline \multirow{3}{*}{$\begin{array}{c}\text { Time of } \\
\text { sampling after } \\
\text { exposure to } \\
14 \mathrm{CO}_{2} \\
\end{array}$} & \multirow{3}{*}{ Plant } & \multirow{3}{*}{$\begin{array}{l}\text { Plant } \\
\text { parts }\end{array}$} & \multicolumn{4}{|c|}{ mg of $\alpha$-amino $N$ of } & \multirow{3}{*}{$\begin{array}{c}\mathrm{mg} \\
\text { of alkaloid } \\
\mathrm{N}\end{array}$} \\
\hline & & & \multicolumn{2}{|c|}{ free } & \multicolumn{2}{|c|}{ protein } & \\
\hline & & & lysine & arginine & lysine & arginine & \\
\hline \multicolumn{8}{|c|}{ Wielkopolski } \\
\hline \multirow{6}{*}{ Immediately } & \multirow{3}{*}{ fodder } & leaves & 0.030 & 0.030 & 2.64 & 1.24 & 0.08 \\
\hline & & & 0.033 & 0.015 & 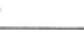 & & 0.09 \\
\hline & & total & 0.063 & 0.045 & & & 0.17 \\
\hline & \multirow{3}{*}{ bitter } & leaves & 0.031 & 0.024 & 3.52 & 2.38 & 2.02 \\
\hline & & stem & 0.016 & 0.017 & & & 1.58 \\
\hline & & total & 0.047 & 0.041 & & & 3.60 \\
\hline \multirow{6}{*}{ After 12 hours } & \multirow{3}{*}{ fodder } & leaves & 0.065 & 0.033 & 2.95 & 0.92 & 0.07 \\
\hline & & stem & 0.024 & 0.028 & 1.19 & 0.54 & 0.08 \\
\hline & & total & 0.089 & 0.061 & 4.14 & 1.46 & 0.15 \\
\hline & \multirow{3}{*}{ bitter } & leaves & 0.035 & 0.037 & 3.35 & 2.70 & 2.02 \\
\hline & & stem & 0.020 & 0.026 & 1.68 & 0.77 & 1.56 \\
\hline & & total & 0.055 & 0.063 & 5.03 & 3.47 & $3.58^{\circ}$ \\
\hline & & & Szybkope & & - & & \\
\hline \multirow{6}{*}{ Immediately } & \multirow{3}{*}{ fodder } & leaves & 0.016 & 0.032 & 2.19 & 0.67 & 0.03 \\
\hline & & stem & 0.020 & 0.016 & & & 0.01 \\
\hline & & total & 0.036 & 0.045 & & & 0.04 \\
\hline & \multirow{3}{*}{ bitter } & leaves & 0.020 & 0.025 & 2.28 & 1.84 & 1.56 \\
\hline & & stem & 0.011 & 0.023 & & & 1.27 \\
\hline & & total & 0.031 & 0.048 & & & 2.83 \\
\hline \multirow{6}{*}{ After 12 hours } & \multirow{3}{*}{ fodder } & leaves & 0.036 & 0.051 & 3.59 & 0.76 & 0.02 \\
\hline & & stem & 0.031 & 0.014 & 1.55 & 0.43 & 0.02 \\
\hline & & total & 0.067 & 0.065 & 5.14 & 1.19 & 0.04 \\
\hline & \multirow{3}{*}{ bitter } & leaves & 0.052 & 0.064 & 3.23 & 2.49 & 1.57 \\
\hline & & stem & 0.008 & 0.021 & 2.32 & 0.74 & 1.26 \\
\hline & & total & 0.060 & 0.085 & 5.55 & 3.23 & 2.83 \\
\hline
\end{tabular}

The relative content of protein lysine in leaves (fig. 1) as well as in stems (fig. 2) of all investigated homozygotes is higher than that of protein arginine. However in no case any significant differences in its content between bitter and fodder plants can be found. On the other hand, these plants show marked differences as regards their protein arginine. The content of this amino acid in leaves of fodder homozygotes is much lower than in bitter ones - in 'Wielkopolski' and especially in 'Szybkopędny'. The stems - organs whose capacity to synthesize alkaloids, in contrast with leaves, is extremely low (at least in L. albus - Birecka 1963) - do not show any differences in this respect. These facts resulted in a much higher lysine/ arginine ratio in leaves of fodder plants than that in bitter ones (in which it amounts to $1.2-1.5$ ); in stems this ratio in both homozygotes is the same amounting in 'Wielkopolski' to about 2.2, in 'Szybkopędny' to 3.2-3.6.

As the percentual content of protein $\alpha$-amino acid $\mathrm{N}$ in fodder plants, especially in leaves is lower than in bitter ones and the content of lysine is the same in both 
of them, the share of this amino acids in the total amount of amino acids is - in contrast with arginine - in the fodder homozygotes higher than in the bitter ones (fig. 3).

The data concerning plant radioactivity immediately after exposure to ${ }^{14} \mathrm{CO}_{2}$ (tab. 3) show some differences in the photosynthetic activity between fodder and

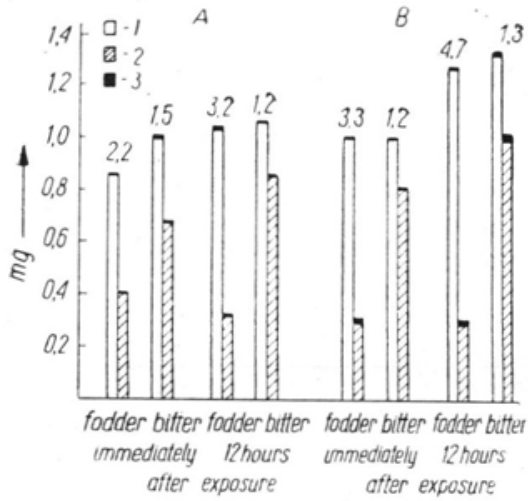

Fig. 1. Lysine and arginine content* in leaves of Lupinus angustifolius - $\mathrm{mg}$ of $\alpha$-amino $\mathrm{N}$ per $\mathrm{g}$ d.m.

$A$ - 'Wielkopolski'; $B$ - 'Szybkopędny'. 1 - protein lysine; 2 - protein arginine; 3 - free lysine or arginine.

* Figures above particular columns indicate the ratio between lysine and arginine.

Fig. 3. The share of lysine and arginine in the total amount of $\alpha$-amino nitrogen. (plants sampled 12 hours after exposure to ${ }^{14} \mathrm{CO}_{2}$ ).

$A$ - 'Wielkopolski'; $B$ - 'Szybkopędny'. 1 - protein lysine in leaves: 2 - protein lysine in stem; 3 - free lysine or arginine; 4 - protein arginine in stem; 5 - protein arginine in leaves

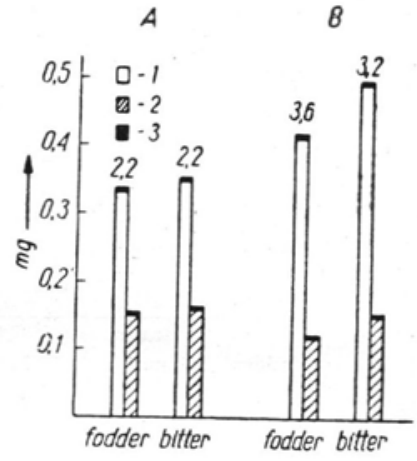

Fig. 2. Lysine and arginine content* in stems of Lupinus angustifolius $-\mathrm{mg}$ of $\alpha$-amino $\mathrm{N}$ per $\mathrm{g}$ d.m. (plants sampled

12 hours after exposure to ${ }^{14} \mathrm{CO}_{2}$ )

A - 'Wielkopolski'; $B$ - 'Szybkopędny'. 1 - protein lysine; 2 - protein arginine; 3 free lysine or arginine.

* Figures above particular columns indicate the ratio between lysine and arginine

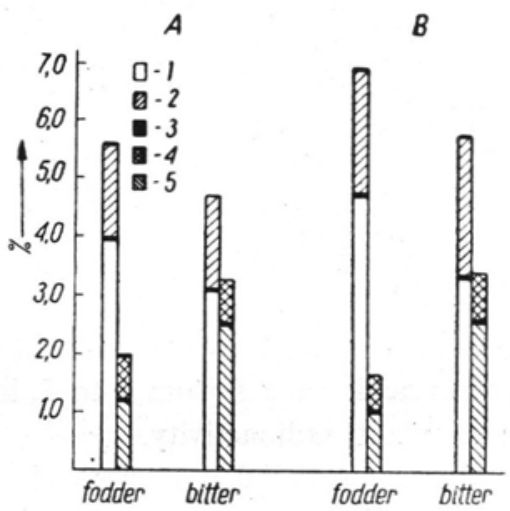

bitter plants - in favor of the latter ones. After 12 hours of darkness, when the aerial parts have lost a considerable amount of their carbon assimilated, the differences between the investigated homozygotes become insignificant. These phenomena, however, require further investigations.

As it could be expected the specific radioactivity of free amino acids immediately after exposure is much higher than of proteins. The differences between fodder 
Table 3

Radioactivity of plants and amino acids (per plant)

\begin{tabular}{|c|c|c|c|c|c|c|c|c|c|}
\hline \multirow{4}{*}{$\begin{array}{l}\text { Time after } \\
\text { exposure } \\
\text { to }{ }^{14} \mathrm{CO}_{2}\end{array}$} & \multirow{4}{*}{ Plant } & \multirow{4}{*}{$\begin{array}{l}\text { Plant } \\
\text { parts }\end{array}$} & \multicolumn{5}{|c|}{ Radioactivity $-10^{3} \mathrm{cpm}$} & \multirow{3}{*}{\multicolumn{2}{|c|}{$\begin{array}{l}\text { Degree of } 14 \mathrm{C} \\
\text { incorporation } \\
-\% \text { of total } \\
\text { radioactivity - } \\
\text { into }\end{array}$}} \\
\hline & & & \multirow{3}{*}{$\begin{array}{c}\text { plant } \\
\text { material } \\
\text { total }\end{array}$} & \multicolumn{4}{|c|}{ total amino acids } & & \\
\hline & & & & \multicolumn{2}{|c|}{$\begin{array}{l}\text { specific activity } \\
\text { per } \mathrm{mM}^{1} \text { ) }\end{array}$} & \multicolumn{2}{|c|}{ total activity } & & \\
\hline & & & & $\begin{array}{c}\text { free } \\
\text { amino } \\
\text { acids }\end{array}$ & $\begin{array}{l}\text { protein } \\
\text { amino } \\
\text { acids }\end{array}$ & $\begin{array}{c}\text { free } \\
\text { amino } \\
\text { acids }\end{array}$ & $\begin{array}{l}\text { protein } \\
\text { amino } \\
\text { acids }\end{array}$ & $\begin{array}{c}\text { free } \\
\text { amino } \\
\text { acids }\end{array}$ & $\begin{array}{c}\text { protein } \\
\text { amino } \\
\text { acids }\end{array}$ \\
\hline \multicolumn{10}{|c|}{ Wielkopolski } \\
\hline \multirow{6}{*}{ Immediately } & \multirow{3}{*}{ fodder } & leaves & 6009 & 945 & 47 & 137 & 173 & 2.3 & 2.9 \\
\hline & & stem & 1896 & 39 & & 6 & traces & 0.3 & \\
\hline & & total & 7905 & & & 143 & & 1.8 & \\
\hline & \multirow{3}{*}{ bitter } & $\begin{array}{l}\text { leaves } \\
\text { stem }\end{array}$ & $\begin{array}{l}7676 \\
2955\end{array}$ & $\begin{array}{r}607 \\
78\end{array}$ & 61 & 156 & 340 & 2.0 & 4.4 \\
\hline & & & 2900 & & & 16 & traces & 0.5 & \\
\hline & & total & 10631 & & & 172 & & 1.6 & \\
\hline \multirow{6}{*}{$\begin{array}{c}\text { After } 12 \\
\text { hours }\end{array}$} & \multirow{3}{*}{ fodder } & leaves & 4745 & 235 & 123 & 58 & 419 & 1.2 & 8.8 \\
\hline & & stem & 2432 & 78 & 20 & 15 & 31 & 0.6 & 1.3 \\
\hline & & total & 7177 & & & 73 & 450 & 1.0 & 6.3 \\
\hline & \multirow{3}{*}{ bitter } & leaves & 3235 & 157 & 108 & 52 & 504 & 1.6 & 15.6 \\
\hline & & stem & 2922 & 157 & 16 & 33 & 40 & 1.1 & 1.4 \\
\hline & & total & 6157 & & & 85 & 544 & 1.4 & 8.8 \\
\hline \multicolumn{10}{|c|}{ Szybkopędny } \\
\hline \multirow{6}{*}{ Immediately } & \multirow{3}{*}{ fodder } & leaves & 6004 & 431 & 80 & 51 & 207 & 0.8 & 3.4 \\
\hline & & stem & 1786 & 59 & & 7 & traces & 0.4 & \\
\hline & & total & 7790 & & & 58 & & 0.7 & \\
\hline & \multirow{3}{*}{ bitter } & leaves & 9701 & 568 & 80 & 121 & 297 & 1.2 & 3.1 \\
\hline & & stem & 2948 & 59 & & 13 & traces & 0.4 & \\
\hline & & total & 12649 & & & 134 & & 1.0 & \\
\hline \multirow{6}{*}{$\begin{array}{c}\text { After } 12 \\
\text { hours }\end{array}$} & \multirow{3}{*}{ fodder } & leaves & 3601 & 255 & 98 & 68 & 320 & 1.9 & 8.9 \\
\hline & & stem & 3319 & 216 & 25 & 31 & 44 & 0.9 & 1.3 \\
\hline & & total & 6920 & & & 99 & 364 & 1.4 & 5.3 \\
\hline & \multirow{3}{*}{ bitter } & leaves & 4201 & 216 & 118 & 58 & 435 & 1.4 & 10.3 \\
\hline & & stem & 3399 & 176 & 18 & 33 & 49 & 1.0 & 1.4 \\
\hline & & total & 7600 & & & 91 & 484 & 1.2 & 6.4 \\
\hline
\end{tabular}

$\left.{ }^{1}\right)$ calculated on the basis of amino $\mathrm{N}$ content.

and bitter plants are not significant. The degree of ${ }^{14} \mathrm{C}$-incorporation into free amino acids ranges from 1 to 2 , into protein amino acids - from 3 to $4 \%$ of the total plant radioactivity.

In plants sampled 12 hours later the ${ }^{14} \mathrm{C}$ incorporation into proteins increased significantly being higher in bitter than in fodder homozygotes.

Almost in all cases the specific activity of free and protein lysine and especially arginine (tab. 4) is much lower than the mean specific activities of total amino acids* (tab. 3).

* These differences cannot be due totally to greater perhaps radioactive impurities in the amino acid solution before chromatographic partition (especially when protein is under consideration). 
Table 4

Radioactivity of lysine, arginine and alkaloids

\begin{tabular}{|c|c|c|c|c|c|c|c|c|c|c|c|c|}
\hline \multirow{4}{*}{$\begin{array}{c}\text { Time } \\
\text { after } \\
\text { exposure } \\
\text { to } \\
{ }^{14} \mathrm{CO}_{2}\end{array}$} & \multirow{4}{*}{ Plant } & \multirow{4}{*}{$\begin{array}{l}\text { Plant } \\
\text { parts }\end{array}$} & \multicolumn{8}{|c|}{ Amino acids } & \multirow{2}{*}{\multicolumn{2}{|c|}{$\frac{\text { Alkaloids } 1 \text { ) }}{\begin{array}{c}\text { radioactivity } 10^{3} \\
\mathrm{cpm}\end{array}}$}} \\
\hline & & & \multicolumn{4}{|c|}{$\begin{array}{l}\text { specific radioactivity } 10^{3} \mathrm{cpm} \\
\text { per } \mathrm{mM} \text { of }\end{array}$} & \multicolumn{4}{|c|}{ total radioactivity $10^{3} \mathrm{cpm}$} & & \\
\hline & & & \multicolumn{2}{|c|}{ free } & \multicolumn{2}{|c|}{ protein } & \multicolumn{2}{|c|}{ free } & \multicolumn{2}{|c|}{ protein } & \multirow{2}{*}{$\mid \begin{array}{c}\text { speci- } \\
\text { fic }^{2} \text { ) } \\
\text { per } \mathrm{mM}\end{array}$} & \multirow{2}{*}{ total } \\
\hline & & & lysine & arginine & lysine & arginine & lysine & arginine & lysine & arginine & & \\
\hline
\end{tabular}

Wielkopolski

\begin{tabular}{|c|c|c|c|c|c|c|c|c|c|c|c|c|}
\hline \multirow{5}{*}{$\begin{array}{l}\text { Imme- } \\
\text { diately }\end{array}$} & \multirow[t]{2}{*}{ fodder } & $\begin{array}{l}\text { leaves } \\
\text { stem }\end{array}$ & $\begin{array}{r}265 \\
49\end{array}$ & $\begin{array}{l}82 \\
39\end{array}$ & 29 & 14 & $\begin{array}{l}0.57 \\
0.12\end{array}$ & $\begin{array}{l}0.18 \\
0.04\end{array}$ & 5.5 & 1.2 & 256 & 1 \\
\hline & & total & & & & & 0.69 & 0.22 & & & & \\
\hline & \multirow{3}{*}{ bitter } & leaves & 184 & 94 & 26 & 17 & 0.41 & 0.16 & 6.6 & 3.0 & 184 & 13.1 \\
\hline & & stem & 120 & 39 & & & 0.14 & 0.05 & & & 43 & 2.4 \\
\hline & & total & & & & & 0.55 & 0.21 & & & & 15.5 \\
\hline \multirow{6}{*}{$\begin{array}{c}\text { After } 12 \\
\text { hours }\end{array}$} & \multirow{3}{*}{ fodder } & leaves & 101 & 147 & 34 & 43 & 0.47 & 0.35 & 7.3 & 2.8 & 370 & 1 \\
\hline & & stem & 45 & 74 & 10 & 5 & 0.08 & 0.15 & 0.9 & 0.2 & 74 & \\
\hline & & total & & & & & 0.55 & 0.60 & 8.3 & 3.0 & & \\
\hline & \multirow{3}{*}{ bitter } & leaves & 116 & 151 & 42 & 40 & 0.29 & 0.37 & 9.0 & 7.8 & 229 & 16.3 \\
\hline & & stem & 129 & 84 & 14 & 20 & 0.18 & 0.16 & 1.7 & 1.1 & 104 & 5.7 \\
\hline & & total & & & & & 0.47 & 0.53 & 10.7 & 8.9 & & 22.0 \\
\hline
\end{tabular}

\begin{tabular}{|c|c|c|c|c|c|c|c|c|c|c|c|c|}
\hline \multicolumn{13}{|c|}{ Szybkopędny } \\
\hline \multirow{5}{*}{$\begin{array}{l}\text { Imme- } \\
\text { diately }\end{array}$} & \multirow[t]{2}{*}{ fodder } & $\begin{array}{l}\text { leaves } \\
\text { stem }\end{array}$ & $\begin{array}{r}252 \\
45\end{array}$ & $\begin{array}{r}174 \\
52\end{array}$ & 40. & 23 & $\begin{array}{l}0.29 \\
0.06\end{array}$ & $\begin{array}{l}0.40 \\
0.05\end{array}$ & 6.2 & 1.1 & & traces \\
\hline & & total & & & & & 0.35 & 0.45 & & & & \\
\hline & \multirow{3}{*}{ bitter } & leaves & 421 & 225 & 35 & 36 & 0.60 & 0.40 & 5.8 & 4.8 & 99 & 8.7 \\
\hline & & stem & 136 & 58 & & & 0.11 & 0.10 & & & 36 & 1.6 \\
\hline & & total & & & & & 0.71 & 0.50 & & & & 10.3 \\
\hline \multirow{6}{*}{$\begin{array}{l}\text { After } 12 \\
\text { hours }\end{array}$} & \multirow{3}{*}{ fodder } & leaves & 177 & 76 & 27 & 40 & 0.45 & 0.28 & 6.9 & 2.2 & 505 & 1 \\
\hline & & stem & 50 & 99 & 7 & 8 & 0.10 & 0.10 & 0.8 & 0.2 & 121 & 1 \\
\hline & & total & & & & . & 0.55 & 0.38 & 7.7 & 2.4 & & \\
\hline & \multirow{3}{*}{ bitter } & leaves & 130 & 88 & 30 & 50 & 0.48 & 0.40 & 6.8 & 8.8 & 327 & 18.0 \\
\hline & & stem & 145 & 80 & 9 & 11 & 0.08 & 0.12 & 1.6 & 0.6 & 104 & 4.6 \\
\hline & & total & & & & & 0.56 & 0.52 & 8.4 & 9.4 & & 22.6 \\
\hline
\end{tabular}

1) The specific activity of alkaloids was determined twice: 1) including hydroxylupanine esters and 2) after their removal. The activity in the latter case was usual lower in leaves, almost the same in stems. The leaves in bitter Wielkopolski contained about 30 and in bitter Szybkopędny $21 \%$ of hydroxylupanine esters (total alkaloid $100 \%$ ); the stems in Wielkopolski -5 and of Szybkopedny $-3 \%$. The reported here radioactivity was calculated on the basis of the specific activity obtained after removal of hydroxylupanine esters (including their amounts).

2) Assuming that the mean molecular weight of alkaloids amounts to 260 .

It is worth stressing that during the two and a half hour exposure to ${ }^{14} \mathrm{CO}_{2}$ a relatively high amount of labelled lysine is formed and already $90 \%$ of its content is to be found in proteins. During the following 12 hours of darkness the radioactivity of this amino acid increased to a rather low degree. The differences between fodder and bitter plants as regards its radioactivity as well as incorporation into proteins are insignificant - either in 'Wielkopolski' or in 'Szybkopędny'. 12 hours after exposure in all investigated homozygotes the radioactivity of bound lysine accounts for about $2 \%$ of the total protein amino acid activity. In contrast 
with lysine the amount of labelled arginine formed during exposure to ${ }^{14} \mathrm{CO}_{2}$ is relatively small; this fact is revealed in its specific as well as total radioactivity in (both - the free and bound - forms). It must be noted that in bitter plants the radioactivity of arginine immediately after exposure is significantly higher than in the fodder ones. These differences become greater after following 12 hours, when the radioactivity of protein arginine in bitter plants increases considerably reaching the level of lysine activity (whereas in fodder plants it remains still three times lower).

The differences in the specific activity of alkaloids in dependence of the sublimation temperature or chromatographic repartition were not very great. The data in table 4 represent the lowest values obtained. Nevertheless one must take account of the occurrence of some* radioactive impurities, especially in alkaloids of fodder plants.

Table 5

Alkaloid content in plants of L. angustifolius var. Wielkopolski gorzki (per 10 plants)

\begin{tabular}{l|l|c|c|c|c|c|c|c}
\hline \multirow{2}{*}{ No. } & \multirow{2}{*}{$\begin{array}{c}\text { Plant age-days } \\
\text { after germination }\end{array}$} & $\begin{array}{c}\text { Mineral } \\
\text { nutrients } \\
\text { applied }\end{array}$ & $\begin{array}{c}\text { aerial } \\
\text { parts }\end{array}$ & root & total & $\begin{array}{c}\text { Fresh weight }-\mathrm{g} \\
\text { aerial } \\
\text { parts }\end{array}$ & root & total \\
\hline 1 & $\begin{array}{l}\text { dry seeds } \\
\text { germinated } \\
\text { seeds }\end{array}$ & & & & $1.931)$ & & & 20.8 \\
\hline 3 & \multirow{2}{*}{10 days } & - & 7.6 & 4.1 & 11.7 & 13.5 & 3.9 & 17.4 \\
\hline 4 & \multirow{2}{*}{25 days } & + & 9.5 & 4.3 & 13.8 & 13.6 & 3.3 & 16.9 \\
\hline
\end{tabular}

1) Dry weight.

Immediatelly after exposure the specific activity or alkaloids in leaves of bitter as well as fodder plants is rather high. Their total radioactivity in bitter homozygotes is about twice as much as that of protein lysine. During the following 12 hours the specific as well as the total radioactivity of alkaloids increases considerably, especially in 'Szybkopędny'.

In both bitter homozygotes the degree of ${ }^{14} \mathrm{C}$ incorporation into alkaloids accounts for $0.35 \%$ of the total labelled carbon assimilated. According to our previous investigations on L. albus (Birecka $1963 \mathrm{a}$, b) such a degree of incorporation indicates a high rate of alkaloid synthesis. It was shown in these investigations than in ten days old white lupin plants, in which no significant alkaloid increment occurs, the synthesis of these bases from new assimilates is minimal. Thus the conclusion was drawn that the rate of alkaloid synthesis in young white lupin plants is very low.

\footnotetext{
* Not very high - according to other investigations carried out in our laboratory.
} 
As the data concerning young bitter plants* of L. angustifolius indicate (tab. $5)^{* *}$ the amount of alkaloids during the first three weeks of growth do not increase significantly, independently on the influence of mineral nutrients (mainly nitrogen) on plant growth.

\section{DISCUSSION}

In the reported here experiments the similarities as well as differences between fodder and bitter plants were analogous in both pairs, 'Wielkopolski' and 'Szybkopędny'.

The first feature to be stressed is the higher relative protein $\alpha$-amino acid $\mathrm{N}$ content in bitter plants, especially in their leaves. It would be interesting to know, whether such difference between fodder and bitter plants (also in other lupin species) is of a casual or typical character.

No significant differences between these plants in the free arginine or lysine content could have been found (at the stage of high rate of alkaloid synthesis!). It seems that their content can not serve as any criterion in discussion on alkaloid precursors. In both investigated homozygotes they constitute a very small proportion $(1-3 \%)$ of the total amount of arginine or lysine; at the same time the total content of alkaloid $\mathrm{N}^{* * *}$ in bitter plants is about the same as the amount of total lysine $\alpha$-amino $\mathrm{N}$ in leaves (whereas in fodder ones it account for $2-6 \%$ only).

The data obtained did not reveal any differences between fodder and bitter plants as regards the total amount of lysine, its specific as well as total radioactivity, differences, which could have been expected. Thus discussed results do not support the lysine hypothesis. However, they should not be considered as a strong argument against it; for one might imagine e.g. such a metabolic situation, in which the rate of synthesis and accumulation of lysine in both, bitter and fodder plants would be similar, but the transformation of a considerable (and the same) proportion of this amino acid would be different: in bitter plants it would give rise to alkaloids, in fodder ones to other compounds. It is true that the results concerning the specific radioactivity of lysine (free and bound) and alkaloids do not support this possibility; but it must be borne in mind that they may or may not represent the true radioactivities at the sites of alkaloid synthesis or lysine transformations within the cell.

However, it should be stressed that the main difference observed between the fodder and bitter plants is revealed in their arginine content, and contrary to what could have been expected according to the arginine hypothesis - the bitter lupins contained more protein arginine than the fodder ones. Is there any relation-

* The variety used also by Mazuś and Reifer (1964).

** The authors are greatly indebted to mgr. St. Głażewski, who helped in carrying out this experiment.

*** According to the lysine hypothesis two $\mathrm{N}$ atoms from three lysine molecules are incorporated into one alkaloid molecule (of a sparteine skeleton). 
ship between these differences and the differences in alkaloid synthesis?* If so, then of what metabolic character? It would be very useful to get more experimental informations needed for answering these questions. It seems that the attempts of Mazuś and Reifer to better understand the role of arginine, among other things, met some difficulties because the rate of alkaloid synthesis at the development stage, at which the plants were used by these authors, was rather low.

It seems worthwhile to examine bitter and fodder plants of other than L. angustifolius lupin species with regard to their basic amino acid composition content and synthesis.

\section{SUMMARY}

Two pairs of homozygotes (each pair - represented by one fodder and one bitter homozygote - had common parents) at the stage of a high rate of alkaloid synthesis were investigated. They showed differences in growth, revealing mainly in the leaf/stem dry weight ratio. The similarities as well as differences between fodder and bitter plants were analogous in both investigated pairs.

The fodder and bitter plants showed the same total content of free amino acids, but differed from each other with regard to the amount of protein $\alpha$-amino $\mathrm{N}$, which was higher in the latter ones. The share of free lysine and arginine varied between 1.1 and $3.1 \%$ of the total content of free amino acids without any differences between fodder and bitter plants. The share of these basic amino acids in proteins was much higher ranging from 7.2 to $10 \%$.

There were no significant differences between investigated varieties as regards the lysine content, its specific as well as total radioactivity. The labelled lysine was formed mainly during the two and a half hours of plant exposure to ${ }^{14} \mathrm{CO}_{2}$. Its increment during the following 12 hours of darkness was relatively small. The leaves of the fodder plants contained - as compared to those of bitter ones - much less protein arginine. The amount of labelled arginine formed during exposure to ${ }^{14} \mathrm{CO}_{2}$ was relatively small, especially in fodder plants. During the following 12 hours the radioactivity of protein arginine in bitter plants increased considerably reaching the level of lysine activity, whereas in fodder plants it remainded still three times lower.

The results obtained are discused in the light of existing hypotheses on alkaloid precursors.

It seems worthwhile to examine bitter and fodder plants of other lupin species with regard to their basic amino acid composition, content and synthesis.

The experiments were supported in part by a research grant form the U.S. Department of Agriculture.

Plant Physiology Department

(Entered: 1.IX.1965)

Agricultural University Warsaw

\section{REFERENCES}

Barbacki S., Mikołajczyk J., Przybylska J., Sulinowski K., 1962, Gen. Pol. 31.

Birecka H., 1963, Acta Soc. Bot. Pol. 32(1):131.

Birecka H., 1963, Bull. Acad. Polon. Sci. ser. sci. biol. 9(7):341.

Birecka H., Rybi cka H., Szymańska W., 1960, Acta Soc..Bot. Pol. 29(2):275.

* It is worth noting that the specific activities of alkaloids in bitter and fodder plants are similar to each other. 
Birecka H., Wojcieska U., 1962, ibidem 31(2):337.

Hasse K., Schmidt G., 1963, Biochem. Z. 337:69.

Hasse K., Schmidt G., 1963, ibidem, 337:480.

Hegnauer R., 1958, Planta Medica, 6:1.

Luckner M., 1964, Die Pharm., 19:9.

Mazuś B., Reifer I., 1964, Bull. Acad. Polon. Sci. ser. sci. biol. 12(7):297.

Mikołajczyk J., 1960, Zesz. Probl. Post. Nauk Roln., 21:389.

Moore S., Stein W. H., 1948, J. Biol. Chem., 176:367.

Mothes K., 1955, Ann. Rev. of Plant Physiol., 6.

Nowacki E., Przy bylska J., Hurich J., 1960, Bull. Acad. Pol. Sci. ser. sci. biol. 8:445.

Nowacki E., Byerrum R. U., 1962, Bioch. Bioph. Res. Comm. 7:58.

Przybylska J., 1959, Bull. Acad. Polon. Sci. ser. sci. biol. 8:362.

Przybylska J. Hurich J., 1960, ibidem, 8:508.

Reifer I., Niziołek S., 1957, Acta Bioch. Pol. 4:3.

Schöpf C., 1948, Angew. Chem. 61:31.

Schöpf C., Arm H., Benz G., 1951, Naturwiss. 38:186.

Schütte H. R., 1960, Arch. Pharm., 293:1006.

Schütte H. R., Nowacki R., 1959, Naturwiss., 46:493.

Schütte H. R., Bohlmann F., Reusche W., 1961, Arch. Pharm. 294:610.

Schütte H. R., Nowacki E., Schäfer Ch., 1962, Arch. Pharm., 295:20.

Schütte H. R., Hindorf H. H., 1964, Naturwiss, 51:463.

Schütte H. R., Hindorf H., Mothes K., Hübner G., 1964, Liebigs Ann. Chem. 93.

Schütte H. R., Lehfeldt J., 1964, Z. Naturforsch, 19(11):1085.

White H. A., Spencer M., 1964, Canad. J. Bot., 42:1481.

Wiewiórowski M., Augustyniak J., Skrzypińska H., Przybylska J., Kociałkowski Z., 1959, Acta Bioch. Pol. 6:143.

Wiewiórkowski M., Reifer I., 1961, Bull. Acad. Polon. Sci. ser. biol. 9:441.

\section{Zasadowe aminokwasy w pastewnych i gorzkich biotypach Lupinus angustifolius}

\section{Streszczenie}

Obiektem badań były dwie pary homozygot łubinu wąskolistnego. Każda para reprezentowana była przez gorzki i pastewny genotyp homologiczny.

W fazie kwitnienia rośliny zdekapitowano i po upływie 10 dni umieszczono je na dwie i pół godziny w atmosferze zawierającej znakowany ${ }^{14} \mathrm{C}$ dwutlenę węgla. Część z nich zebrano natychmiast po ekspozycji, pozostałe po 12 godzinach (w ciemności).

Rośliny zabito w temp. $-60^{\circ} \mathrm{C}$ i w tejże temperaturze rozdrobniono. Materiał roślinny został przeanalizowany na zawartość oraz radioaktywność aminokwasów wolnych i związanych w białkach, ze szczególnym uwzględnieniem lizyny i argininy.

Zaobserwowane podobieństwa i różnice między roślinami pastewnymi i gorzkimi były analogiczne w obu badanych parach homozygot.

Rośliny pastewne i gorzkie wykazywały jednakową (i jednocześnie b. niską) zawartość wolnych aminokwasów, ale różniły się pod względem zawartości aminokwasów białkowych. Zawartość tych ostatnich była wyższa w homozygotach gorzkich. Udział wolnej lizyny i argininy w ogólnej ilości wolnych aminokwasów wahał się w granicach od 1.1 do $3.1 \%$. Nie stwierdzono różnic pod tym względem między roślinami gorzkimi i pastewnymi. Udział lizyny i argininy w białkach był znacznie wyższy stanowiąc 7.2 do $10 \%$ ogólnej ilości $\alpha$-amino N. 
Rośliny pastewne i gorzkie nie wykazywały żadnych istotnych różnic ani pod względem zawartości lizyny, ani też pod względem jej radioaktywności właściwej i ogólnej; ${ }^{14} \mathrm{C}$-lizyna tworzyła się głównie w czasie ekspozycji roślin na ${ }^{14} \mathrm{CO}_{2}$; w ciągu następnych 12 godzin jej ilość wzrosła w stosunkowo małym stopniu;

Liście roślin pastewnych - w porównaniu z liśćmi roślin gorzkich - zawierały znacznie mniej argininy. Ilość ${ }^{14} \mathrm{C}$-argininy bezpośrednio po ekspozycji była względnie mała, szczególnie w homozygotach pastewnych. W ciągu następnych 12 godzin radioaktywność argininy białka w roślinach gorzkich znacznie wzrosła osiągając poziom radioaktywności lizyny, podczas gdy w roślinach pastewnych pozostawała jeszcze trzykrotnie niższa.

Uzyskane wyniki dyskutowane są w świetle istniejących hipotez odnośnie do prekursorów alkaloidów.

Wydaje się celowe przebadanie roślin pastewnych i gorzkich innych gatunków łubinu pod względem składu aminokwasów zasadowych, ich zawartości i syntezy.

Katedra Fizjologii Roślin

SGGW

Warszawa 